\title{
As dificuldades de percepção do enfermeiro da atenção primária à saúde do homem
}

\author{
Simone Maria de A. Maia, ${ }^{1 *}$ Willian Malagutti $^{2}$
}

\begin{abstract}
Resumo
Introdução: Segundo descrito na Política Nacional de Atenção Integral da Saúde do Homem (PNAISH) os homens têm dificuldade em reconhecer suas necessidades, cultivando o pensamento mágico que rejeita a possibilidade de adoecer. A política de saúde do homem existe, mas é pouco discutida. A justificativa desse estudo se baseia no fato que no Brasil, embora a expectativa de vida dos homens tenha aumentado, segundo dados do IBGE, de 59,7 para 69,7 anos entre 1980 e 2010, desde 1991 ela vem se mantendo 7,6 anos abaixo da média das mulheres. Objetivos: Apontar as dificuldades de percepção do enfermeiro da atenção primária à saúde do homem e discutir sobre a forma que tais dificuldades podem interferir em sua prática. Métodos: Trata-se de pesquisa bibliográfica, qualitativa, de caráter descritivo-exploratório. Resultados: Com a discussão das publicações potenciais, duas categorias emergiram: a atuação do enfermeiro na Estratégia Saúde da Família (ESF) e as dificuldades do enfermeiro em atuar na saúde do homem. O estudo discute estratégias dentro do que se espera na PNAISH. Conclusões: Existem estratégias que podem ser consideradas ao acolher indivíduos do sexo masculino na ESF e o enfermeiro é um facilitador para que sejam alcançados resultados satisfatórios.
\end{abstract}

Descritores: Enfermagem em saúde comunitária; Saúde do homem; Saúde da família.

\section{Abstract \\ The difficulties of perception of primary health nurse towards men's health}

Introduction: As described in the National Comprehensive Care of Human Health Policy (PNAISH) in Brazil, there is the difficulty in men to recognizing their needs, cultivating a magical thinking that rejects the possibility of becoming ill. The man's health policy exists, but it is also little discussed. The rationale of this study is because in Brazil, although the life expectancy of men has increased, according to IBGE data, from 59.7 to 69.7 years between 1980 and 2010, since 1991 it has remained 7.6 years below the woman average. Objectives: To indicate the difficulties of perception of nurses of primary health of men and discuss how such difficulties can interfere with your practice. Method: This is a bibliographic, qualitative, descriptive and

Exploratory research study. Results: From the discussion of potential publications, two categories emerged: the action of nurses in the Family Health Strategy (ESF) and the difficulties of nurses at work in men's health. The study discusses strategies within what PNAISH expects. Conclusions: There are
1. Universidade Anhanguera Educacional (UNIAN), Niterói, Rio de Janeiro, Brasil.

2. Universidade de Brasília, Brasília, Brasil.

\author{
*Endereço para correspondência: \\ Universidade Anhanguera Educacional \\ Rua Zoraida Brasil Alcantara, 38, Ap 202 \\ Niterói, RJ. Brasil. CEP: 24130-135. \\ E-mail: simoneamaia@yahoo.com.br
}

Revista HUPE, Rio de Janeiro, 2016;15(4):336-342

doi: 10.12957/rhupe.2016.31611

Recebido em 02/08/2016. Aprovado em 07/09/2017.

strategies that can be considered in order to embrace this man and the nurse is a facilitator for achieving satisfactory results. Keywords: Community health nursing; Men's health Family health.

\section{Resumen}

Las dificultades de percepción de lo enfermero de la primaria de salud para la salud del hombre

Introdución: Como se describe en la Política Nacional de Atención Integral de Salud del Hombres (PNAISH) en Brasil, existe

la dificultad de los hombres a reconocer sus necesidades, cultivando el pensamiento mágico que rechaza la posibilidad de enfermarse. Existe una política de salud del hombre, pero es poco discutida. La razón fundamental de este estúdio está dada por el hecho de que en Brasil, aunque la esperanza de vida de los hombres ha aumentado, según los datos del IBGE, de 59,7 a 69,7 años entre 1980 y 2010, desde 1991 se há mantenido 7.6 años siguientes a la de media de las mujeres. Objetivos: Señalar las dificultades de percepción de las enfermeras de la salud primaria del hombre y discutir en que estas dificultades pueden interferir con su práctica. Método: El estudio forma parte del modelo de investigación bibliográfica, cualitativo, descriptivo y exploratorio. Con la discusión de publicaciones potenciales surgido dos categorías: el papel de las enfermeras en la Estrategia Salud de la Familia (ESF) y las dificultades de las enfermeras en el trabajo en la salud del hombre. El estudio analiza las estrategias dentro de lo que se espera de la PNAISH. Resultados: Hay estrategias que se pueden considerar para acoger este hombre y la enfermeira es un facilitador para que los resultados ocurren de maneira satisfatória. 
Palabras clave: Enfermeira em salud comunitaria; Salud del hombre; Salud de la familia.

\section{Introdução}

A Estratégia Saúde da Família (ESF) é uma das principais propostas preconizadas pelo Ministério da Saúde do Brasil, para reorientar o modelo assistencial do Sistema Único de Saúde, a partir da atenção básica dentro de uma perspectiva de estabelecimento de maior vínculo, criação de laços de compromisso e coparticipação entre profissionais e comunidade. ${ }^{1}$

A família é o foco de atenção. Ela é vista de forma integral a partir de onde mora. Nesse espaço é onde se constroem relações e vínculos para uma compreensão do processo saúde/doença e intervenções ativas para a melhora em sua qualidade de vida. ${ }^{1}$

O enfermeiro tem um papel importante dentro da ESF, o que tem representado um reconhecimento social deste profissional, pela sua atuação ativa como política integrativa, holística e humanizadora.

A Portaria 648/2006 do Ministério da Saúde; ${ }^{2}$ referente à Política Nacional da Atenção Básica; traz as atribuições mínimas dos enfermeiros da ESF. São atribuições de suma importância para a implementação desta estratégia como tática de reorganização do primeiro nível de atenção à saúde.

A atuação da enfermagem vem se expandindo, estando atualmente presente em diferentes processos de trabalho, desde hospital geral, Centro Psicossocial de Atenção e Ambulatórios e a Estratégia Saúde da Família (ESF), serviços estes localizados dentro dos espaços coletivos e norteados pelo conceito ampliado de saúde, com base na concepção de gerenciamento do cuidado. ${ }^{3}$

O enfermeiro integra a equipe da ESF como coordenador e deve estar preparado para o enfrentamento e a resolução de problemas que correspondam à saúde da mulher, da criança e adolescente, do adulto e idoso, saúde do homem, assim como o controle de diabetes, hipertensão, tuberculose, hanseníase e saúde bucal. ${ }^{3}$

No ano de 2009, o Ministério da Saúde lançou também a Política Nacional de Atenção Integral à Saúde do Homem (PNAISH), com o objetivo de facilitar e ampliar o acesso da população masculina aos serviços de saúde. ${ }^{4}$

A PNAISH promove ações de saúde respeitando as diferentes maneiras de ser dos homens, organizando-se através de três eixos principais: saúde sexual, reprodutiva e paternidade, violências e acidentes em geral e o acesso ou acolhimento dos homens na atenção básica, porta de entrada prioritária na Rede SUS.

Segundo descrito na PNAISH, existe a dificuldade dos homens em reconhecer suas necessidades, cultivando o pensamento mágico que rejeita a possibilidade de adoecer.

O nosso interesse em abordar esse tema através de levantamento bibliográfico de publicações sobre o assunto surgiu como inquietação do papel do enfermeiro nesta problemática. Existiam questionamentos e discussões sobre as diferentes áreas da ESF exceto sobre a saúde do homem. Além de ter havido pouca abordagem das estratégias que poderiam ter sido criadas pelo profissional enfermeiro como educador, dentro desta estratégia política, desde o ano em que a política foi criada.

A política de saúde do homem existe, mas é pouco discutida. No Brasil, embora a expectativa de vida dos homens tenha aumentado, segundo dados do IBGE, de 59,7 para 69,7 anos entre 1980 e 2010, desde 1991 ela vem se mantendo 7,6 anos abaixo da média das mulheres.

Como problema norteador tem-se a indagação de quais as dificuldades de percepção do enfermeiro da atenção primária à saúde do homem. Mediante o exposto, os objetivos do presente artigo são apontar as dificuldades de percepção do enfermeiro da atenção primária à saúde do homem e discutir sobre de quais formas tais dificuldades podem interferir em sua prática.

\section{Entendendo a política nacional de atenção integral à saúde do homem}

Nos 20 anos do Sistema Único de Saúde (SUS), o Ministério da Saúde (MS) apresenta uma das prioridades do governo, a Política Nacional de Atenção Integral à Saúde do Homem, desenvolvida em parceria entre gestores dos SUS, sociedades científicas, sociedade civil organizada, pesquisadores, acadêmicos e agências de cooperação internacional. ${ }^{4}$

A política traduz um longo anseio da sociedade ao reconhecer que os agravos do sexo masculino constituem verdadeiros problemas de saúde pública. ${ }^{4}$

Para isso, a Política Nacional de Atenção Integral à Saúde do Homem trabalha em conjunto com a Política Nacional de Atenção Básica - porta de entrada do Sistema Único de Saúde - com as estratégias de humanização, e em consonância com os princípios do SUS, fortalecendo ações e serviços em redes e cuidados da saúde. O Ministério da Saúde vem cumprir seu papel ao formular a Política que deve nortear as ações de atenção integral à saúde do homem, estimulando o autocuidado 


\section{Artigo de revisão}

e, sobretudo, o reconhecimento de que a saúde é um direito social básico. ${ }^{4}$

Mobilizar a população masculina brasileira pela luta e garantia de seu direito social à saúde é um dos desafios dessa política. Ela pretende tornar os homens protagonistas de suas demandas, consolidando seus direitos de cidadania. ${ }^{4}$

\section{Dados científicos}

A Tabela 1 apresenta as fontes de consultas sobre a temática abordada, com os objetivos de cada autor. Os autores se preocupam em reconhecer a atuação do enfermeiro na trajetória da ESF como facilitador para todas as políticas de saúde que existem. Em oposição a isto, apresentam as dificuldades que impedem o avançar desse profissional. ${ }^{3-12}$

\section{Metodologia}

O estudo se enquadra no modelo de pesquisa bibliográfica, qualitativa, de caráter descritivo-exploratório, pois, embora não tenhamos a finalidade de estabelecer soluções práticas para os problemas enfocados, pretendemos colaborar, a partir de uma reflexão teórico-prática, com o campo da assistência pública de saúde. ${ }^{13-14}$

Utilizou-se a forma exploratória para investigação da realidade e construção da pesquisa, pois essa fase proporciona uma aproximação maior com o campo de observação para melhor delinearmos outras questões como construção da pesquisa. ${ }^{15}$

O levantamento de dados foi realizado através de produções publicadas nos últimos dez anos, entre 2008 a 2017. Todo este processo supracitado foi realizado em uma pesquisa on-line na Biblioteca Virtual de Saúde (BVS) e no Google Acadêmico.

Realizou-se o levantamento bibliográfico em artigos sobre o assunto e, após, foi realizada uma síntese em alguns dos materiais encontrados. Em levantamento prévio, para realizar as discussões, as temáticas convergentes foram agrupadas, das quais emergiram as seguintes categorias: a atuação do enfermeiro na ESF e as dificuldades do enfermeiro em atuar na saúde do homem.

\section{Resultados e discussão de dados}

Segue a discussão das bibliografias potenciais com as seguintes categorias:

\section{A atuação do enfermeiro na ESF}

A educação em saúde faz parte do cotidiano do enfermeiro, é a forma de sensibilizar, criar vínculos e motivar a população dando a ela o empoderamento nas suas decisões em querer mudar ou não a seu estilo de vida. No modelo tradicional, ela ainda é repleta de ações relacionadas à mudança de pensamento e de comportamento das pessoas, sem se atentar para a influência que os fatores socioeconômicos, espirituais e culturais têm sobre o processo saúde/doença dos indivíduos. Portanto, as ações educativas em saúde precisam ser realizadas com o objetivo de integrar a comunidade nos serviços, demonstrando a ela a importância da sua participação efetiva nas decisões junto à equipe que possa determinar os objetivos e as propostas de trabalho para com a própria comunidade, visando à promoção da saúde. ${ }^{10}$

A agenda de trabalho também é um instrumento significativo para uma boa organização do serviço. A ESF é uma política de saúde que tem importante potencial de ação sobre as demandas dos adolescentes, por estar bem próximo da realidade sociocultural das comunidades, dispondo assim de instrumentos que facilitam o cumprimento dos princípios que norteiam o SUS. 5

Atualmente, com a Portaria Ministerial 648/2006, ${ }^{2}$ a denominação ficou mais abrangente, nomeando as ações de controle de hipertensão, planejamento familiar, entre outras de Saúde do Adulto, o que parece ainda não resolver esta fragmentação da assistência, em que o usuário parece ser ainda visto e atendido em muitos casos como o hipertenso, o diabético, ou seja, de acordo com sua patologia.

Aos profissionais da ESF, cabe programar ações de planejamento familiar para que estes direitos sejam verdadeiramente usufruídos pela população alvo. Compete aos enfermeiros, portanto, assistir à população em termos de concepção e contracepção e fornecer informações sobre os métodos legais e disponíveis no país, despertando, sobretudo, para a importância de uma vivência sexual responsável e saudável, sem negar o acesso aos métodos naturais e comportamentais, tomando por base o dispositivo de lei do planejamento familiar (Lei $\mathrm{n}^{\circ}$ 9.263/96) do Ministério da Saúde. ${ }^{1}$

O controle das endemias faz parte importante das atividades dos enfermeiros que atendem as diretrizes da promoção e proteção à saúde, preconizadas à rede básica. Dentre essas ações mínimas previstas para as equipes da ESF estão as voltadas para o controle da tuberculose e hanseníase.

A pesquisa identifica homens que frequentam a unidade da atenção primária nas seguintes categorias: homens entre 30 e 50 anos em suas atividades laborais ativas, sendo minoria e com pouco tempo disponível 
Simone Maria de Araújo Maia, Willian Malagutti. • As dificuldades de percepção do enfermeiro da atenção primária à saúde do homem

Tabela 1. Levantamento bibliográfico sobre a atuação do enfermeiro na trajetória da Estratégia da Saúde da Família (ESF).

\begin{tabular}{|c|c|c|c|}
\hline Código & Título do artigo & Autor/Ano & Objetivo do artigo \\
\hline A1 & $\begin{array}{l}\text { A prática do enfermeiro na Estratégia Saúde da } \\
\text { Família: o caso do município de Vitória/ES }\end{array}$ & $\begin{array}{l}\text { Silva VG, Motta } \\
\text { MCS, Zeitoune } \\
\text { RCG, } 2010\end{array}$ & $\begin{array}{l}\text { Caracterizar a prática do enfermeiro na Estratégia } \\
\text { Saúde da Família }\end{array}$ \\
\hline $\mathrm{A} 2$ & $\begin{array}{l}\text { A atuação do enfermeiro em educação em saúde: } \\
\text { uma perspectiva para a atenção básica }\end{array}$ & $\begin{array}{l}\text { Gonçalves GG, } \\
\text { Soares M, } 2010\end{array}$ & $\begin{array}{l}\text { Descrever os aspectos gerais da educação em } \\
\text { saúde; evidenciando os obstáculos e facilidades } \\
\text { encontrados pelos enfermeiros para que seja } \\
\text { desenvolvida tal ação }\end{array}$ \\
\hline A3 & O enfermeiro em suas diversas áreas de atuação & $\begin{array}{l}\text { Grenzel JCM, et } \\
\text { al, } 2011\end{array}$ & $\begin{array}{l}\text { Conhecer os perfis do enfermeiro em saúde pública } \\
\text { do enfermeiro hospitalar }\end{array}$ \\
\hline A4 & $\begin{array}{l}\text { Atuação do enfermeiro como educador no pro- } \\
\text { grama saúde da família: importância para uma } \\
\text { abordagem integral na atenção primária }\end{array}$ & $\begin{array}{l}\text { Amaral LR et al, } \\
2011\end{array}$ & $\begin{array}{l}\text { Identificar a importância deste profissional como } \\
\text { educador no PSF }\end{array}$ \\
\hline A5 & $\begin{array}{l}\text { Política nacional de atenção integral à saúde do } \\
\text { homem (princípios e diretrizes) }\end{array}$ & Brasil, 2008 & $\begin{array}{l}\text { Promover a melhoria das condições de saúde da } \\
\text { população masculina do Brasil }\end{array}$ \\
\hline A6 & $\begin{array}{l}\text { A atenção à saúde do homem: ações e perspectivas } \\
\text { dos enfermeiros }\end{array}$ & $\begin{array}{l}\text { Santana EN, et } \\
\text { al, } 2010\end{array}$ & $\begin{array}{l}\text { Oferecer subsídios à organização dos serviços de- } \\
\text { senvolvidos no âmbito da ESF e ao planejamento } \\
\text { de ações de promoção à saúde que contemplem } \\
\text { a população masculina }\end{array}$ \\
\hline A7 & $\begin{array}{l}\text { Promoção da saúde do adolescente: uma estraté- } \\
\text { gia de educação em saúde mediante círculos de } \\
\text { cultura }\end{array}$ & $\begin{array}{l}\text { Neto WB et al, } \\
2008\end{array}$ & $\begin{array}{l}\text { Desenvolver estratégias de Educação em Saúde } \\
\text { mediante círculos de cultura com os adolescentes }\end{array}$ \\
\hline A8 & $\begin{array}{l}\text { Nova política de atenção quer levar o "sexo forte" } \\
\text { aos postos }\end{array}$ & Brasil, 2008 & $\begin{array}{l}\text { Levar o universo da literatura das áreas da saúde } \\
\text { à TV e à web }\end{array}$ \\
\hline A9 & $\begin{array}{l}\text { Educação em saúde na estratégia saúde da família: } \\
\text { o significado e a práxis dos enfermeiros }\end{array}$ & $\begin{array}{l}\text { Roecker S, Mar- } \\
\text { con SS, } 2011\end{array}$ & $\begin{array}{l}\text { Identificar o significado da educação em saúde } \\
\text { para os enfermeiros da estratégia saúde da família } \\
\text { e as ações educativas individuais e coletivas desen- } \\
\text { volvidas por eles }\end{array}$ \\
\hline A10 & $\begin{array}{l}\text { Atividades desenvolvidas por enfermeiros no PSF } \\
\text { e dificuldades } m \text { romper o modelo flexneriano }\end{array}$ & $\begin{array}{l}\text { Silva SA et al, } \\
2011\end{array}$ & $\begin{array}{l}\text { Conhecer quais são as atividades e principais di- } \\
\text { ficuldades enfrentadas pelos enfermeiros do PSF }\end{array}$ \\
\hline A11 & $\begin{array}{l}\text { A Atuação do Enfermeiro na Equipe de Saúde da } \\
\text { Família e a Satisfação } \\
\text { Profissional }\end{array}$ & $\begin{array}{l}\text { Araújo MFS, Ol- } \\
\text { iveira FMC, } 2009\end{array}$ & $\begin{array}{l}\text { Analisar a atuação e a satisfação profissional dos } \\
\text { enfermeiros do Programa Saúde da Família }\end{array}$ \\
\hline A12 & $\begin{array}{l}\text { A visão dos profissionais sobre a presença e as } \\
\text { demandas dos homens nos serviços de saúde: per- } \\
\text { spectivas para a análise da implantação da Política } \\
\text { Nacional de Atenção Integral à Saúde do Homem }\end{array}$ & $\begin{array}{l}\text { Knauth DR et al, } \\
2012\end{array}$ & $\begin{array}{l}\text { Analisar as concepções que os profissionais da } \\
\text { saúde possuem sobre as demandas e os com- } \\
\text { portamentos específicos da população masculina } \\
\text { atendida nos serviços de saúde. }\end{array}$ \\
\hline A13 & $\begin{array}{l}\text { Saúde do homem e masculinidades na Política } \\
\text { Nacional de Atenção Integral à Saúde do Homem: } \\
\text { uma revisão bibliográfica }\end{array}$ & $\begin{array}{l}\text { Separavich MA, } \\
\text { Canesqui AM, } \\
2013\end{array}$ & $\begin{array}{l}\text { Analisar da saúde do homem e masculinidades na } \\
\text { PNAISH, à luz da reflexão que estes temas rece- } \\
\text { beram nos últimos tempos na literatura da Saúde } \\
\text { Coletiva/Saúde Pública. }\end{array}$ \\
\hline A14 & $\begin{array}{l}\text { Atenção à saúde do homem: trabalhando a per- } \\
\text { cepção do profissional enfermeiro na estratégia } \\
\text { saúde da família }\end{array}$ & $\begin{array}{l}\text { Siqueira EL et al, } \\
2014\end{array}$ & $\begin{array}{l}\text { Investigar a percepção do enfermeiro na Estratégia } \\
\text { Saúde da Família acerca da atenção à saúde do } \\
\text { homem e das ações desenvolvidas para a imple- } \\
\text { mentação da mesma. }\end{array}$ \\
\hline A15 & $\begin{array}{l}\text { Promoção da saúde do homem: uma experiência } \\
\text { exitosa na atenção básica }\end{array}$ & $\begin{array}{l}\text { Pereira JC et al, } \\
2015\end{array}$ & $\begin{array}{l}\text { Disseminar uma ferramenta para integração en- } \\
\text { tre ensino-serviço-comunidade, a partir de uma } \\
\text { experiência exitosa na criação de um grupo de } \\
\text { Saúde do Homem. }\end{array}$ \\
\hline A16 & $\begin{array}{l}\text { Conflito em equipe: desafios enfrentados por } \\
\text { acadêmicos de enfermagem }\end{array}$ & $\begin{array}{l}\text { Feldhaus C et al, } \\
2016\end{array}$ & $\begin{array}{l}\text { Descrever a vivência de acadêmicos de enfer- } \\
\text { magem em campo de prática em uma ESF, onde se } \\
\text { observou a existência de conflitos entre a equipe } \\
\text { de saúde. }\end{array}$ \\
\hline A17 & $\begin{array}{l}\text { Promoção a saúde do homem na atenção primária } \\
\text { a saúde: um relato de experiência }\end{array}$ & $\begin{array}{l}\text { Silva EAL et al, } \\
2017\end{array}$ & $\begin{array}{l}\text { Relatar a experiência do projeto de ensino e } \\
\text { extensão para a promoção da saúde do homem } \\
\text { trabalhador. }\end{array}$ \\
\hline
\end{tabular}




\section{Artigo de revisão}

ao serviço de saúde. Já na segunda categoria, os idosos já com comorbidades instaladas como hipertensão arterial e/ou diabetes. ${ }^{17}$

O Ministério da Saúde lançou em 2002 o Plano de Reorganização da Atenção à hipertensão arterial e ao diabetes mellitus, sendo indispensável que os municípios adotem essas ações dentre as estratégias mínimas.

O número de jovens que utilizam drogas vasoativas orais (Cialis, Viagra, Levitra) vem aumentando com o objetivo de obter um desempenho sexual diferenciado, e esteroide anabólico para adquirir um corpo "perfeito", colocando em risco suas vidas e saúde. ${ }^{18}$ Percebe-se, portanto, a importância de ações de saúde voltadas para a captação desses jovens citados na pesquisa.

A supervisão é um instrumento de gestão de programas de saúde. O Ministério da Saúde preconiza que o(a) enfermeiro(a) instrutor(a)/supervisor(a) deve desenvolver ações específicas na coordenação e supervisão dos Agentes Comunitários da Saúde (ACS), além daquelas que rotineiramente desenvolve coordenando a Unidade Básica de Saúde. Muitas são as atividades que mantêm relação com a coordenação dos ACS, consideradas atribuições básicas do(a)s enfermeiro(a)s instrutor(a)/(es)/supervisor(a)/(es).

As dificuldades do enfermeiro em atuar na saúde do homem

Atualmente nos serviços de saúde existem dificuldades relacionadas ao fato de que a maioria das ações é planejada pelos coordenadores de equipes e pelos gestores municipais sem que haja a participação dos cidadãos, mesmo tendo claro que as ações serão voltadas ao atendimento exclusivo da população da área de abrangência, ou seja, normalmente as ações são planejadas mediante metas e objetivos da ESF e não conforme as reais necessidades de saúde de determinada população. $^{10}$

Segundo o estudo de Silva, Motta e Zeitoune, ${ }^{5}$ o enfermeiro participa do planejamento do cuidado à saúde das famílias. Porém, avaliando do ponto de vista do processo de trabalho das equipes de saúde da família, este é um instrumento pouco aplicado, por despender muito tempo na construção, análise e atuação frente às exigências de cuidados encontradas, e também na sua atualização, visto que é o retrato de um momento do ciclo vital da família, processo inerentemente dinâmico.

Ainda um dos resultados deste estudo foi que o tempo gasto com ações de prevenção e promoção da saúde é significativamente menor do que o gasto em atividades de gerenciamento e assistência desenvolvi- das nas Unidades de Saúde, e que o horário preferencial para estas atividades é o período da manhã.

As mulheres são a maioria da população brasileira (50,77\%), sendo as principais usuárias do SUS, segundo o Ministério da Saúde. Isso ocorre provavelmente devido à criação do Programa de Assistência Integral à Saúde da Mulher (PAISM) em 1984, o qual foi ao encontro das necessidades de saúde deste retrato social que está mais vulnerável ao adoecimento por diversas razões. ${ }^{16}$

Na sua implantação, o PAISM estimulou que a assistência fosse para além das especificidades biológicas, e trouxe a reflexão sobre a importância da assistência voltada para a compreensão das desigualdades nas condições de vida, nas relações entre homens e mulheres, a sobrecarga de trabalho e a responsabilidade pelo trabalho doméstico e de criação dos filhos, além de empenharem-se nos problemas associados à sexualidade e à reprodução, as dificuldades relacionadas à anticoncepção, a prevenção de doenças sexualmente transmissíveis. Assim, com a implantação das equipes de Saúde da Família, essas ações foram atribuídas dentre as prioritárias para a estratégia.

Porém as políticas públicas de saúde para os adolescentes, regulamentadas desde década de 80 , se desenvolveram aparentemente de forma fragmentada e desarticulada, dificultando até os dias atuais a promoção de ações intersetoriais que garantam a integralidade da assistência.

As disparidades demandam ações de saúde específicas para os homens em todos os níveis da atenção. A Política Nacional de Atenção Integral à Saúde do Homem, lançada em 2009, pelo Ministério da Saúde, visa reunir forças para ampliar o acesso da população masculina aos serviços de saúde, contribuindo para a redução das causas de morbidade e mortalidade e a organização de uma rede de atenção à saúde que garanta uma linha de cuidados integrais para a redução da morbimortalidade, cuja porta de entrada é a Estratégia da Saúde da Família, com ações comunitárias. ${ }^{16}$

Resultados em uma pesquisa apresentaram pouco conhecimento dos enfermeiros em relação à política direcionada para os homens. ${ }^{19}$

Muitos agravos poderiam ser evitados caso os homens realizassem, com regularidade, as medidas de prevenção primária. A resistência masculina à atenção primária aumenta não somente a sobrecarga financeira da sociedade, mas também, e, sobretudo, o sofrimento físico e emocional do paciente e de sua família na luta pela conservação da saúde e da qualidade de vida dessas pessoas. 
Grande parte da não adesão às medidas de atenção integral, por parte do homem, decorre das variáveis culturais.

Uma questão apontada pelos homens para a não procura pelos serviços de saúde está ligada a sua posição de provedor. Alegam que o horário do funcionamento dos serviços coincide com a carga horária do trabalho. Não se pode negar que na preocupação masculina a atividade laboral tem um lugar destacado, sobretudo em pessoas de baixa condição social, o que reforça o papel historicamente atribuído ao homem de ser responsável pelo sustento da família.

A compreensão das barreiras socioculturais e institucionais é importante para a proposição estratégica de medidas que promovam o acesso dos homens aos serviços de atenção primária, a fim de resguardar a prevenção e a promoção como eixos necessários e fundamentais de intervenção. ${ }^{4}$

Existem estratégias que podem ser discutidas em reuniões e articuladas entre as equipes na atenção primária com aplicações práticas para o público-alvo discutido. Conforme resultados de um estudo de 2015, fizeram primeiramente uma reunião, na qual foram identificados e apresentados planos de ação, com escolhas de temas a serem abordados com os usuários. Nesse encontro foi acordado que cada equipe faria o levantamento do número de homens de suas áreas. A execução das atividades ocorreu de forma exitosa, com ajuda de estudantes, além de parceiros de equipamentos sociais. Foram elas: encontro realizado às $19 \mathrm{~h}$, periodicidade mensal, duração aproximada de 1 hora, recepção com música, vídeos educativos, rodas de conversa e apresentação de artistas locais com objetivo de sensibilizar o homem a se empoderar da sua própria saúde. O envolvimento da equipe interdisciplinar facilitou os serviços oferecidos a esses homens. ${ }^{20}$

Observou-se através desta citação acima, evidentes dificuldades, mas as ações podem acontecer de forma sincronizada e resolutiva, permitindo, assim, que os envolvidos obtenham uma produção gratificante, pessoal e profissional.

\section{Considerações finais}

Os resultados deste estudo apresentam entraves fundamentais na realização das atividades dos enfermeiros dentro da ESF e das suas dificuldades cotidianas. A partir deste estudo, espera-se contribuir no campo de atuação na ESF, incentivando os profissionais de saúde, em particular o enfermeiro, a desenvolver trabalhos educativos diferenciados, ou seja, focados nas necessida- des da população e em parceria com ela, transformando as ações em práticas concisas, eficazes e eficientes, capazes de contribuir com a promoção da saúde.

O conflito em equipe e a falha na comunicação são trazidos em pesquisas como fatores que prejudicam o bom andamento dos resultados. ${ }^{21}$ Por outro lado, o entrosamento entre as equipes facilita estratégias relevantes no trabalho. foram feitas visitas a locais estratégicos como: oficinas, metalúrgicas, bares, lavajatos, mercados, marcenarias, construções, pontos de mototáxi e fábricas, a fim de estabelecer vínculo e apresentar serviços oferecidos pela unidade de saúde mais próxima. Processo de trabalho estabelecido entre equipe e colaboradores. ${ }^{22}$

Por fim, percepções podem ser considerados ao acolher esse homem. São elas: conhecer a PNAISH e treinar os ACS, mapear as necessidades do homem na comunidade onde mora, ouvi-lo, preparar o cenário (unidade de saúde) com cartazes sobre saúde do homem, não deixar este cenário tão feminino, incentivá-los a participar do pré-natal de sua companheira e daí aproveitarem para a captação desse homem, planejar grupos educativos dentro de um horário flexível e com parcerias, ir até o homem e não apenas esperá-lo vir até nós. E o enfermeiro é um facilitador para que os resultados aconteçam.

\section{Referências}

1. Brasil. Ministério da Saúde. Regula o $\S 7^{\circ}$ do art. 226 da Constituição Federal, que trata do planejamento familiar, estabelece penalidades e dá outras providências. Lei $n^{\circ}$ 9.263, de 12 de janeiro de 1996. Acesso em 31/08/17.

2. Brasil. Ministério da Saúde. Portaria $n^{\circ} 2.488$, de 21 de outubro de 2011. Aprova a Política Nacional de Atenção Básica, estabelecendo a revisão de diretrizes e normas para a organização da Atenção Básica, para a Estratégia Saúde da Família (ESF) e o Programa de Agentes Comunitários de Saúde (PACS). Diário Oficial [da República Federativa do Brasil], Brasília, n.204, p.55, 24 out. 2011 . Seção 1, pt1.

3. Amaral LR, Oliveira MAD, Cardoso RB, et al. Atuação do enfermeiro como educador no programa saúde da família: importância para uma abordagem integral na atenção primária. FG Ciência. 2011;19(1):1-21.

4. Brasil. Ministério da Saúde. Secretaria de Atenção à Saúde Departamento de Ações Programáticas Estratégicas - Política Nacional de Atenção Integral à Saúde do Homem: Princípios e diretrizes, Brasília, 2008.

5. Silva VG, Motta MCS, Zeitoune RCG. A prática do enfermeiro na Estratégia Saúde da Família: o caso do município de Vitória/ ES. Rev Eletr Enf [on-line]. 2010;12(3):441-448.

6. Gonçalves GG, Soares M. A atuação do enfermeiro em educação em saúde: uma perspectiva para a atenção básica. CUC Salesiano. 2010; p.14. Disponível em http://www.unisalesiano. edu.br/biblioteca/monografias/51937.pdf

7. Grenzel JCM, Cavalheiro DJ, Lazzari B, et al. O enfermeiro em suas diversas áreas de atuação. UNICRUZ. 2011;p.1. 
8. Santana EM, Lima EMM, Bulhões JLF, et al. A atenção à saúde do homem: ações e perspectivas dos enfermeiros. Rev. Min. de Enf. 2010;15(3):324-332.

9. Neto WB, Gomes IMB, Brady CL, et al. Promoção da saúde do adolescente: uma estratégia de educação em saúde mediante círculos de cultura. In: XVI mostra de iniciação científica, Pernambuco. 2008.

10. Roecker S, Marcon SS. Educação em saúde na estratégia saúde da família: o significado e a práxis dos enfermeiros. Esc Anna Nery. 2011;15(4):701-709.

11. Silva SA, Oliveira F, Spinola CM, et al. Atividades desenvolvidas por enfermeiros no PSF e dificuldades em romper o modelo flexneriano. Rev. Enferm. Cent. O. Min. 2011;1(1):30-39.

12. Araujo MFS, Oliveira FMC. A Atuação do Enfermeiro na Equipe de Saúde da Família e a Satisfação Profissional. Revista Eletrônica de Ciências Sociais. 2009;14(1):03-14.

13. Minayo MCS. O desafio do conhecimento: pesquisa qualitativa em saúde. 4. ed. São Paulo, 1996. Intervalo de páginas pp-pp.

14. Gil AC. Como elaborar projetos de pesquisa. São Paulo: Editora Atlas, $5^{a}$ edição, 2010.

15. Deslandes FS. Análise do discurso oficial sobre humanização da assistência hospitalar. Ciência e saúde Coletiva. 2003;9(1):7-14.

16. Brasil. Ministério da Saúde. Secretaria de Atenção à Saúde Departamento de Ações Programáticas Estratégicas - Política Nacional de Atenção Integral à Saúde da Mulher: plano de ação 2004-2007, Brasília. 2004.

17. Knauth DR, Couto MT, Figueiredo WS. A visão dos profissionais sobre a presença e as demandas dos homens nos serviços de saúde: perspectivas para a análise da implantação da Política Nacional de Atenção Integral à Saúde do Homem. Ciência \& Saúde Coletiva. 2012;17(10):2617-2626. Disponível em: http:// www.redalyc.org/html/630/63024360011/

18. Separavich MA, Canesqui AM. Saúde do homem e masculinidades na Política Nacional de Atenção Integral à Saúde do Homem: uma revisão bibliográfica. Saúde Soc. São Paulo, v.22, n.2, p.415-428, 2013. Acesso em 31/08/17.

19. Siqueira EL, Oliveira GR, Mendes JD, et al. Atenção à saúde do homem: trabalhando a percepção do profissional enfermeiro na estratégia saúde da família. Sanare-revista de políticas públicas, v. 13, n. 1, 2014. Disponível em: https://sanare. emnuvens.com.br/sanare/article/view/432

20. Pereira JC, Rocha LTS, Albuquerque SGE, et al. Promoção da saúde do homem: uma experiência exitosa na atenção básica. Rev. APS. 2015 jan/mar; 18(1):123-126. Acesso em 31/08/17.

21. Feldhaus C, Fontana RB, Rutke TCB, et al. Conflito em equipe: desafios enfrentados por acadêmicos de enfermagem. Salão do Conhecimento. 2016;2(2). Acesso em 31/08/17.

22. Silva EAL, Santiago AS, Santos JS, et al. Promoção a saúde do homem na atenção primária a saúde: um relato de experiência. Revista de APS. 2017;19(4). Disponível em: https://aps.ufjf. emnuvens.com.br/aps/article/view/2808. Acesso em 31/08/17. 